\section{Type of Gradient Recalled-Echo Sequence Results in Size and Number Change of Cerebral Microbleeds}

Microbleeds (MBs) are thought to be clusters of hemosiderin-containing macrophages and are more commonly observed in hypertensive microangiopathy (lacunar infarct or intracerebral hemorrhage), cerebral amyloid angiopathy, or cerebral autosomal dominant arteriopathy with subcortical infarcts and leukoencephalopathy (CADASIL) but not exclusively in them. ${ }^{1}$ Although they are usually defined as the focal signal intensity loss of less than 5 or $10 \mathrm{~mm}$, the conditions of gradient recalled-echo (GRE) varied among numerous previous studies about MBs (ie, TEs, were from $15 \mathrm{~ms}$ to $50 \mathrm{~ms}$ ). ${ }^{2,3}$ We found that MBs are influenced in size and number by lengthening TE of GRE, as seen in the following case.

The GRE (TR, $700 \mathrm{~ms}$; TE, $17 \mathrm{~ms}$; flip angle, 20 $0^{\circ}$ of an elderly hypertensive woman with right thalamic hemorrhage and multiple lacunar infarcts showed numerous MBs in the basal ganglia, thalami, pons, cerebellum, and cerebral white matter (Fig 1). The sizes and numbers of these MBs increased as the TE was lengthened (Fig 1). The numbers of MBs in this figure were 12 (TE, $10 \mathrm{~ms}$ ), 14 (TE, $17 \mathrm{~ms}$ ), and 20 (TE, $23 \mathrm{~ms}$ ). The average diameter of MBs, which was $2.6 \mathrm{~mm}$ with a TE of $10 \mathrm{~ms}$, became 3.8 (TE, $17 \mathrm{~ms}$ ) and $4.5 \mathrm{~mm}$ (TE, $23 \mathrm{~ms}$ ).

In general, the $\mathrm{T} 2^{\star}$-based signal intensity loss increases mainly by lengthening TE and decreasing the flip angle. ${ }^{4}$ To the best of our knowledge, no one has reported how the appearance of MBs changes with these parameters. As we have shown, MBs changed considerably in size and number as TE was prolonged. Therefore, the MR imaging parameter must be taken into account, especially when discussing the number or size of MBs. ${ }^{2,3}$ GRE with longer TE raises sensitivity of MBs, but the pathophysiological significance is to be clarified in the future.

\section{References}

1. Koennecke HC. Cerebral microbleeds on MR imaging: Prevalence, associations, and potential clinical implications. Neurology 2006;66:165-71

2. Fazekas F, Kleinert R, Roob G, et al. Histopathologic analysis of foci of signa intensity loss on gradient-echo $\mathrm{T} 2{ }^{*}$-weighted images in patients with spontaneous intracerebral hemorrhage: evidence of microangiopathy-related microbleeds. AJNR Am J Neuroradiol 1999;20:637-42

3. Greenberg SM, Eng JA, Ning MM, et al. Hemorrhage burden predicts recurrent intracerebral hemorrhage after lobar hemorrhage. Stroke 2004;35: 1415-20

4. Mulkern RV. Fast imaging principles. In: Atlas SW, ed. MR Imaging of the Brain and Spine, 3rd ed. Philadelphia: Lippincott Williams \& Wilkins; 2002:176-78

S. Tatsumi

T. Ayaki

M. Shinohara

T. Yamamoto

Department of Neurology

Saiseikai Nakatsu Hospital and Medical Center

Osaka, Japan

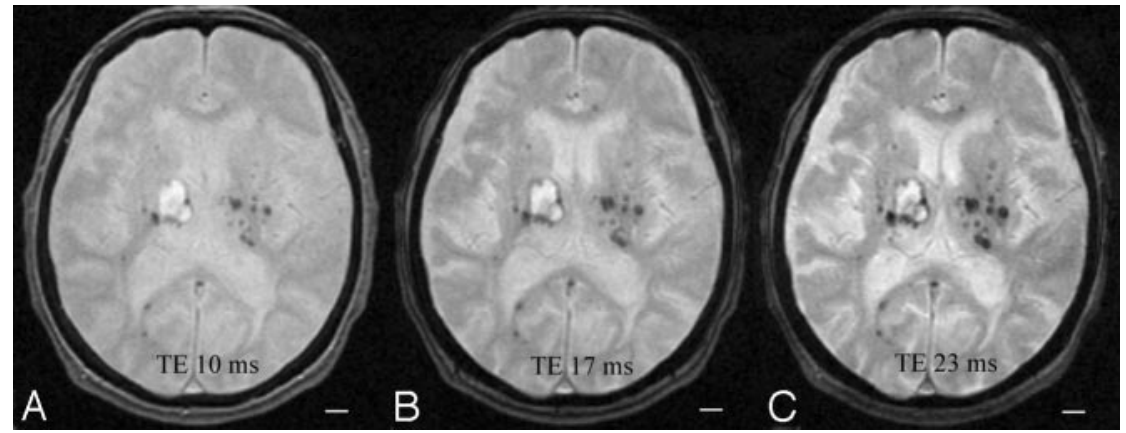

Fig 1. The size and number of microbleeds increased by lengthening TE on GRE. Scale bar, $10 \mathrm{~mm}$. 